\title{
Estimating Combined Correlation Functions for Dipoles in Rayleigh-Fading Scenarios
}

\author{
Juan F. Valenzuela-Valdés, Antonio M. Martínez-González, and David Sánchez-Hernández, Senior Member, IEEE
}

\begin{abstract}
While the spatial correlation function between two dipoles is well known, the angular correlation function for different arbitrary inclination angles in transmission and reception is not available in the literature. In this paper, a novel angular correlation function is proposed for two dipoles with arbitrary inclination angles between transmission and reception, which is useful for true polarization diversity schemes. Likewise, two original functions for estimating combined spatial-angular correlation are proposed. The novel functions are used with previously reported results and validated for Rayleigh-fading scenarios with isotropic scattering using a reverberation chamber.
\end{abstract}

Index Terms-Dipole, multiple-input-multiple-output (MIMO), polarization diversity, reverberation chamber, spatial diversity.

\section{INTRODUCTION}

$\mathbf{T}$ HE correlation between different multiple-input-multipleoutput (MIMO) channels can be used to estimate the performance of the MIMO system since it is the result of interaction of the scattering environment and antenna properties. Losses due to correlation are quantified in [1]. Mutual coupling effects on the correlation coefficients are studied in [2] and three-dimensional (3-D) models for obtaining the correlation coefficients are described in [3]. In many studies, the correlation factor is employed to study MIMO system performance by assuming that the correlation among receive antennas is independent from the correlation between transmit antennas, that is, with no transmit-receive cross-correlation. This simplification makes a separable and analytically friendly model. When the antennas have the same radiation patterns, this model is known as the Kronecker model. When the correlation occurs only at either the receiver or transmitter ends, but not at both, the model is known as semicorrelated [4]. Some other models with cross-correlation assumptions are also available [5], including some analyses of the capacity and symbol error rate errors [6]. Some recent studies, however, claim that in order to obtain a good estimation of MIMO performance, it is essential to consider the relationship between transmit and receive antennas [7].

In a previous paper [8], we have studied the effect of true polarization diversity, writing an equivalence between spatial and true angular diversity. Based upon this equivalence, we hereby propose an estimation of the angular correlation function for two dipoles that are rotated a certain arbitrary angle respect to the

Manuscript received March 15, 2007; revised May 8, 2007. This work was supported in part by Fundación Séneca, Autonomous Region of Murcia, Spain, under Projects 2I05SU0033 and TIC-TEC 06/01-0003.

The authors are with the Departamento de Tecnologías de la Información y Comunicaciones, Technical University of Cartagena, Cartagena E-30202, Spain (e-mail: david.sanchez@upct.es; http://www.gimre.upct.es).

Digital Object Identifier 10.1109/LAWP.2007.900962

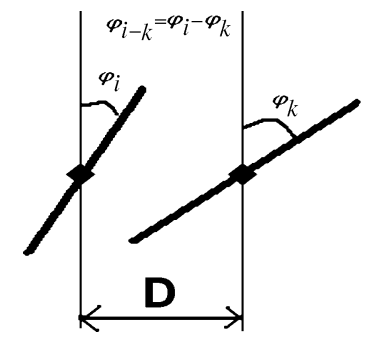

Fig. 1. Geometry of the proposed dipole antenna correlation function.

other using part of the spatial-correlation function. The novel function is validated for an isotropic environment in which all angles of arrival (AoA) are possible, namely, with an angular spread of $360^{\circ}$. With the novel estimation of an angular correlation function, we also propose an original function for combining diversity, that is, a function that is capable of estimating the correlation between two dipoles that are spatially separated by a distance $d$ and angularly separated by a rotation angle $\varphi$, one respect to the other, as depicted in Fig. 1.

The novel simulated results are validated by both comparison to previously reported simulation results and measurements using a reverberation chamber. Despite initial chambers' being prepared to emulate Rayleigh-fading environments with some polarization imbalance, it has been demonstrated recently that both polarization performance [9] and Rician-fading scenarios [10] can properly be emulated in reverberation chambers, therefore extending their emulating capabilities to indoor and scenarios with a dominant line-of-sight component. In this letter, the models are validated by measurement in the Bluetest $A B$ RC800 reverberation chamber with an isotropic environment.

Isotropic scattering can be considered for indoor scenario modeling when a great number of scatters are present. When metallic windows and other artifacts, trees, and walls in buildings are incorporated in the model, the richer multipath environment is very similar to an isotropic scattering [11]. Some works [12] provide models with a dependence on the number of scatters, obtaining several relationships between the number of multipath components and the Rician K-factor or the MIMO capacity. It is therefore possible to encounter in practice the isotropic scattering for indoor environments that will be used in this letter to validate the proposed functions.

\section{INDIVIDUAL CORRELATION FUNTIONS}

\section{A. Spatial Correlation Model}

The spatial correlation between two adjacent identical antennas due to the arrival of a single plane wave can be approximated by an exponential function of its incident angle [13]. 
The autocorrelation coefficients between two vertically polarized antennas in the idealized two-dimensional (2-D) Clarke isotropic scattering environment are obtained by $J_{o}(k d)$, where $k$ is wave number, $d$ the space separation, and $J_{o}(k d)$ the zeroorder Bessel function of the first kind [14]. The spatial correlation function for dipole antennas in a multipath environment was originally developed by [15] and later validated using the reverberation chamber for isotropic environments [16] by

$$
\zeta=\sin \left[z_{\text {spatial }}\right] /\left[z_{\text {spatial }}\right]
$$

where $z_{\text {spatial }}=2 * \pi * d_{\text {spatial }} / \lambda$ and $d_{\text {spatial }}$ is the interelement distance. However, existing 2-D or isotropic spatial correlation functions involve important differences to full 3-D auto- or cross-correlation functions, and its careful use with practical implementations is recommended [17]. Consequently, 3-D correlation models have been called for. Recently, far-field formulas have been suggested to calculate the complex crosscorrelation coefficients of dipole antennas in a 3-D model, which includes the effects of mutual coupling [2], [3]. Yet, even for this 3-D model, results are only valid for cross-polarization ratios XPR $=1$, that is, for conventional orthogonal polarization diversity. The recent development of true-polarization diversity schemes, wherein the full potential of polarization diversity can be exploited by using a progressive angular rotation between elements [8], makes the isotropic spatial correlation model and the 3-D far-field correlation model insufficient to accurately predict the novel correlation scenario, and new correlation functions are needed.

\section{B. Angular Correlation Model}

In a true polarization scheme, the angular difference between two dipoles is defined as

$$
\varphi_{i-j}=\varphi_{i}-\varphi_{j}
$$

where $\varphi_{i}$ and $\varphi_{j}$ are the orientation angles of the dipoles. By representing an angular separation in true polarization diversity as equivalent to the spatial separation distance, a new correlation function can be defined by

$$
\zeta=\sin \left[z_{\text {angular }}\right] /\left[z_{\text {angular }}\right]
$$

where $z_{\text {angular }}=2 * \pi * d_{\text {angular }}$. The angular separation $d_{\text {angular }}$ for Rayleigh-fading scenarios with isotropic scattering can be made equivalent to a spatial separation by

$$
d_{\text {angular }}=\varphi_{i-j}^{\prime} / 180 .
$$

In this model, the angular difference between two consecutive dipoles is transformed to an angle of the first quadrant in the following way:

$\varphi_{i-j}^{\prime}=\left\{\begin{array}{c}a b s\left(\varphi_{i-j}\right) \text { for } a b s\left(\varphi_{i-j}\right) \leq 90 \\ 180-a b s\left(\varphi_{i-j}\right) \text { for } 90 \leq a b s\left(\varphi_{i-j}\right) \leq 270 \\ 360-a b s\left(\varphi_{i-j}\right) \text { for } 270 \leq a b s\left(\varphi_{i-j}\right) \leq 360\end{array}\right\}$.

It is worth mentioning here that the model is only valid when the angular displacement between dipoles exists only in one axis. Multiaxis true angular diversity, recently proposed for

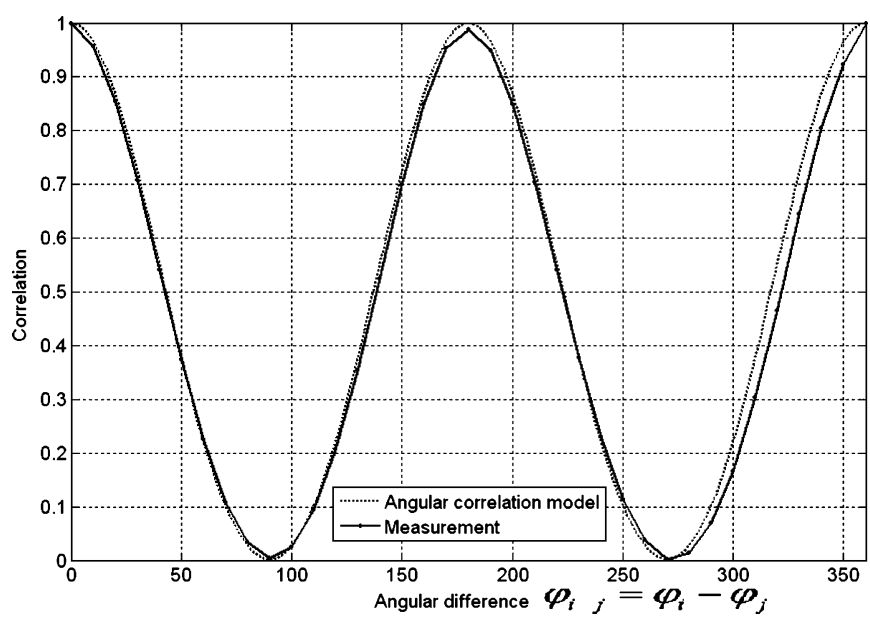

Fig. 2. Simulated and measured angular correlation model versus angular difference of two dipoles.

three monopoles in a handset [18], is therefore not accounted for in this letter.

\section{Measurement Results}

In order to validate the new angular correlation function, measurements of the correlation matrix of two dipoles were performed in the RC800 reverberation chamber. All measurements illustrated in this letter have been performed with the RC800 reverberation chamber by Bluetest $A B$, with dimensions of $0.8 \times 1 \times 1.6 \mathrm{~m}$, three wall-mounted antennas, 25 platform stirring positions, three polarization stirring positions due to three different orthogonal wall-mounted printed-antenna exciting elements, two mechanical stirrers with 15 different positions for each platform position, and $20 \mathrm{MHz}$ frequency stirring. Measurements were performed at $900 \mathrm{MHz}$ and two half-wave dipoles were used as MIMO array antennas. Thirty-six different measurements were carried out by rotating one dipole up to $360^{\circ}$ in $10^{\circ}$ steps respect to the other dipole.

Both simulated and measured coefficients of the new correlation function are depicted in Fig. 2. Good matching between the new model and measurements is observed. It seems clear that, when using dipoles, there is a periodic pattern and a symmetry within the period of the correlation function, so that only $0-90^{\circ}$ angular differences need to be simulated. An error $<0.05$ in the simulation of the correlation coefficient was observed.

\section{COMBINED CORRELATION FunCTIONS}

The full potential of the novel correlation function can be exploited when combined to other spatial correlation functions. Two different ways are proposed and validated to combine spatial and angular diversity models.

\section{A. Linear Combined Model}

The combined effect provides a complex combined correlation that has not been studied in depth yet. Should the spatial correlation coefficient simply be added to the angular correlation coefficient, nonrealistic situations with correlation coefficients higher than one would be made possible. Else, the combined correlation would increase instead of the expected 


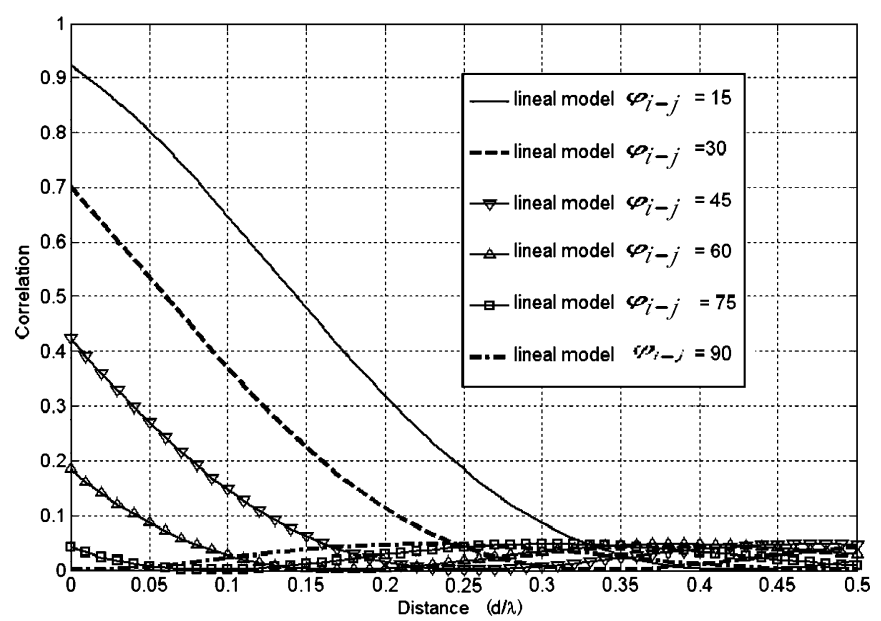

Fig. 3. Lineal model for combined correlation function versus spatial distance with angular difference as parameter.

decrease since nonidentical orientations are used. Consequently, we propose a linear model which merges the spatial distance with its equivalent distance for angular diversity in a linear manner by

$$
\zeta=\sin \left[z_{\text {total }}\right] /\left[z_{\text {total }}\right]
$$

where $Z_{\text {total }}=Z_{\text {spatial }}+Z_{\text {angular. }}$. In Fig. 3, the correlation for the linear combined model is depicted with spatial distances ranging from $0.1 \mathrm{~d} / \lambda$ to $0.5 \mathrm{~d} / \lambda$ and equivalent angular distances between $15-90^{\circ}$. As happened to the diversity gain in [8], it is clear that when there is a large spatial separation, true polarization diversity can hardly reduce the already low correlation coefficient. In contrast, and also in a similar way to what happened for diversity gain in [8], when short spatial distances are employed, true polarization diversity can be very useful for reducing the high correlation coefficients. With just two dipoles, the best option is to employ orthogonal polarizations, that is, an angular difference of $90^{\circ}$ between them.

\section{B. Quadratic Combined Model}

Since the angular separation has been used as an equivalent distance to spatial separation [8], the combination of the distances for obtaining $z_{\text {total }}$ to be used in (6) can also be performed in a quadratic way by

$$
z_{\text {total }}=\sqrt{z_{\text {spatial }}^{2}+z_{\text {angular }}^{2}} \text {. }
$$

The correlation coefficients for this model are depicted in Fig. 4 for the same spatial and angular ranges employed in Fig. 3. Figs. 3 and 4 confirm, this time for the correlation coefficients, the duality between true angular and spatial diversity demonstrated in [8] for the diversity gain.

The reduction in the correlation coefficient as a consequence of increasing the spatial separation of two dipoles with true angular diversity is similar to the reduction obtained by incrementing the angular separation of two dipoles already separated by a fixed spatial distance. As an example, a spatial separation of $\sim 0.2 \lambda$ is equivalent to an angular separation of $\sim 36^{\circ}$. The presented combined correlation function models can therefore be

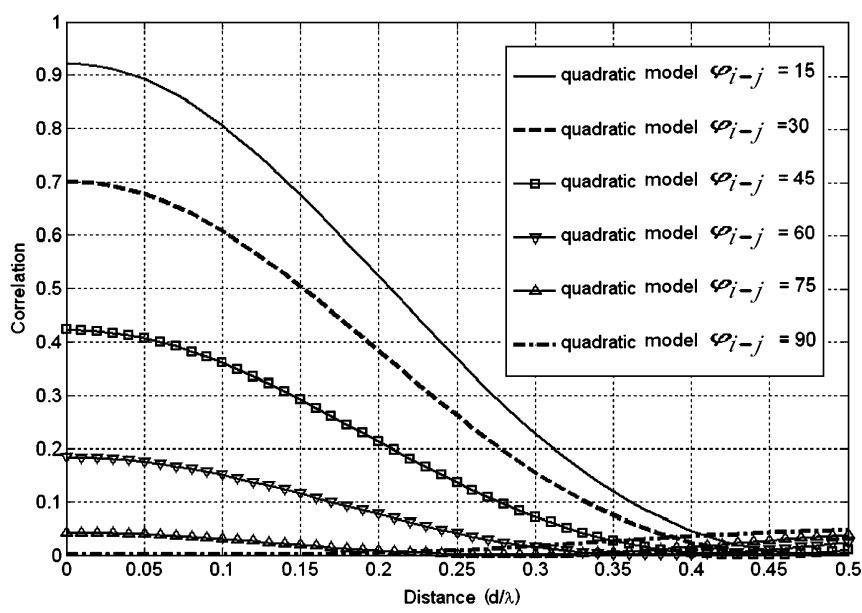

Fig. 4. Quadratic model for combined correlation function versus spatial distance with angular difference as parameter.

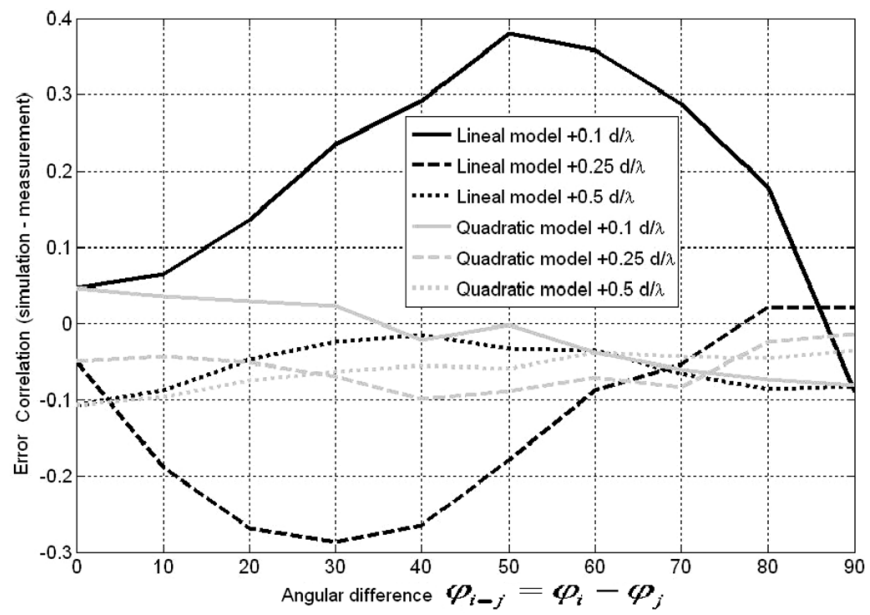

Fig. 5. Errors in combined models for the correlation functions versus angular difference with distance as a parameter.

useful for deriving true-polarization diversity figures when combined to spatial-diversity techniques. There are, however, some differences between the two proposed combined models, and a validation through simulations and measurements is required.

\section{Measurement Results}

Validation measurements were carried out using the RC800 reverberation chamber. A comparison between measurements and the simulations using both the linear and the quadratic combined models is illustrated in Figs. 5 and 6, wherein the encountered differences are reproduced versus angular and spatial separation distances, respectively.

A careful observation of Figs. 5 and 6 reveals that the linear combined model does not work well when there is a variation in the angular separation. Yet, while from Fig. 5 it seems that the quadratic model has a moderate and quasi-constant error respect to a variation in the angular separation, from Fig. 6 one can also observe that this behavior is not maintained when the spatial separation is altered. In fact, the linear- and quadratic-combined models seem to follow opposite error behaviors when the spatial separation is altered. In both Figs. 5 and 6, the maximum error 


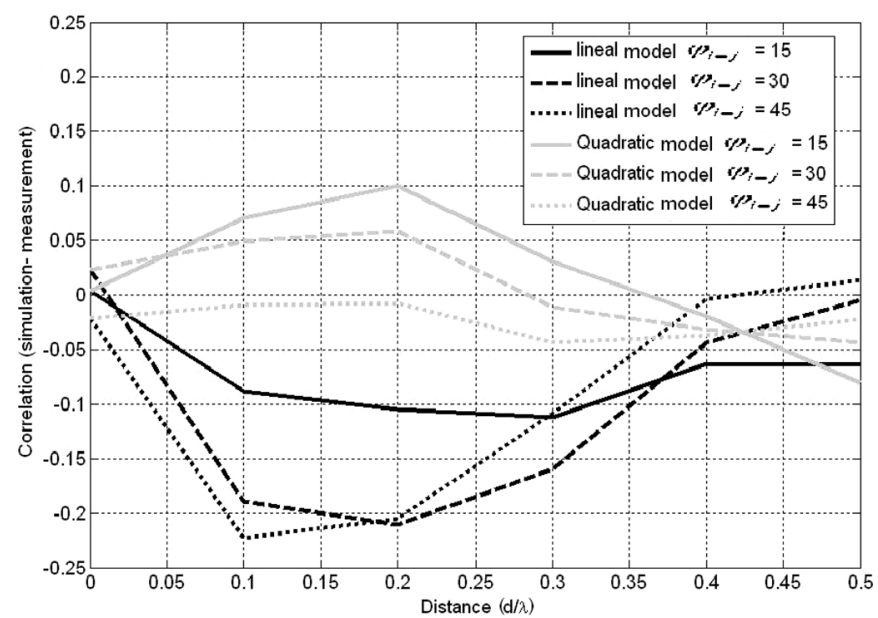

Fig. 6. Errors in combined models for the correlation functions versus spatial separation with angular distance as a parameter.

in the quadratic model $(0.10)$ is lower than the maximum error of the linear model $(0.22)$, but other higher order combining possibilities or the use of weighting factors are possible, and further research is envisaged. These errors are similar to other previously reported spatial-only models [3].

\section{CONCLUSION}

In this letter, we have presented a novel angular correlation function for two dipoles to be employed with true-polarization schemes. The new function has also been successfully combined to conventional spatial correlation functions so that hybrid spatial- and true-polarization diversity schemes can be properly evaluated. The new combined functions have been validated through measurements in a reverberation chamber. The new functions are useful for Rayleigh-fading environments with isotropic scattering and its extension to other fading scenarios is envisaged. Since many MIMO models employ correlation functions to evaluate system performance and since hybrid spatialand true-polarization diversity measurements are cumbersome, the new functions can be considered an easy and powerful tool for practical design considerations of novel MIMO systems in handheld terminals and base stations.

\section{REFERENCES}

[1] X. Li and Z. Nie, "Performance losses in V-BLAST due to correlation," IEEE Antennas Wireless Propag. Lett., vol. 3, no. 1, pp. 291-294, 2004.

[2] A. Derneryd and G. Kristensson, "Signal correlation including antenna coupling," Electron. Lett., vol. 40, no. 3, pp. 157-159, Feb. 2004

[3] P. Hui, C. G. Hynes, J. V. Wonterghem, and D. G. Michelson, "3-D autocorrelation coefficients of dipole antenna," Electron. Lett., vol. 42, no. 5, pp. 257-258, Mar. 2006.

[4] M. Kang and M.-S. Alouni, "Capacity of correlated MIMO Rayleigh Channels," IEEE Trans. Wireless Commun., vol. 5, no. 1, pp. 143-155, Jan. 2006.

[5] A. M. Tulino, A. Lozano, and S. Verdu, "Impact of antenna correlation on the capacity of multiantenna channels," IEEE Trans. Inf. Theory, vol. 51, no. 7, pp. 2491-2509, Jul. 2005.

[6] C. Oestges, B. Clerckx, D. Vanhoenacker-Janvier, and A. J. Paulraj, "Impact of fading correlations on MIMO communication systems in geometry-based statistical channel models," IEEE Trans. Wireless Commun., vol. 4, no. 3, pp. 1112-1120, May 2005.

[7] W. Weichselberger, M. Herdin, H. Ozcelik, and E. Bonek, "A stochastic MIMO channel model with joint correlation of both link ends," IEEE Trans. Wireless Commun., vol. 5, no. 1, pp. 90-100, Jan. 2006.

[8] J. F. Valenzuela-Valdés, M. A. García-Fernández, A. M. MartínezGonzález, and D. Sánchez-Hernández, "The role of polarization diversity for MIMO systems under Rayleigh-fading environments," IEEE Antennas Wireless Propag. Lett., vol. 5, pp. 534-536, 2006.

[9] P. S. Kildal and C. Carlsson, "Detection of a polarization imbalance in reverberation chambers and how to remove it by polarization stirring when measuring antenna efficiencies," Microw. Opt. Technol. Lett., vol. 34, no. 2, pp. 145-149, Jul. 2002.

[10] C. L. Holloway, D. A. Hill, J. M. Ladbury, P. F. Wilson, G. Koepke, and J. Coder, "On the use of reverberation chambers to simulate a Rician radio environment for the testing of wireless devices," IEEE Trans. Antennas Propag., vol. 54, no. 11, pp. 3167-3177, Nov. 2006.

[11] Z. Yun and M. F. Iskander, "MIMO capacity for realistic wireless communications environments," in Proc. IEEE Antennas Propag. Soc. Int. Symp., Jun. 2004, pp. 1231-1234.

[12] C. Oestges, V. Erceg, and A. J. Paulraj, "A physical scattering model for MIMO macrocellular broadband wireless channels," IEEE J. Sel. Areas Commun., vol. 21, pp. 721-729, Jun. 2003.

[13] Z. Tang and A. S. Mohan, "Characterize the indoor multipath propagation for MIMO communications," in Proc. Asia-Pacific Microw. Conf. (APMC), Dec. 2005, vol. 4, p. 4.

[14] R. H. Clarke, "A statistical theory of mobile radio reception," Bell Syst. Tech. J., vol. 47, pp. 957-1000, 1968.

[15] W. C. Jackes, Microwave Mobile Communications. New York: Wiley, 1974.

[16] D. Hill and J. M. Ladbury, "Spatial/correlation functions of fields and energy density in a reverberation chamber," IEEE Trans. Electromagn. Compat., vol. 44, pp. 95-101, Feb. 2002.

[17] P. D. Doncker, "Spatial correlation functions for fields in three-dimensional Rayleigh channels," in Proc. Progr. Electromagn. Res. Symp. (PIER), 2003, vol. 40, pp. 55-69.

[18] L. Dong, H. Choo, R. W. Heath, and H. Ling, "Simulation of MIMO channel capacity with antenna polarization diversity," IEEE Trans. Wireless Commun., vol. 4, pp. 1869-1873, Jul. 2005. 